\title{
Ring imaging Cherenkov counter of HERMES for pion, kaon, proton and anti-proton identification
}

\author{
Toshi-Aki Shibata \\ Tokyo Institute of Technology, Tokyo, Japan
}

\section{for the HERMES collaboration}

\section{A R T I C L E I N F O}

Available online 5 May 2014

Keywords:

$\mathrm{RICH}$

Dual-radiator

Aerogel

Deep inelastic scattering experiment

HERMES

\begin{abstract}
A B S T R A C T
RICH of HERMES was built for identification of pion, kaon, proton and anti-proton in the momentum range of 2-15 GeV/c. It was a dual-radiator $\mathrm{RICH}$. The radiators were aerogel and $\mathrm{C}_{4} \mathrm{~F}_{10}$ gas. Produced hadrons in electron-nucleon deep inelastic scattering were identified by the RICH and spin structure of the nucleon was studied by correlation between the directions of the target spin, scattered electron and produced hadrons.
\end{abstract}

(c) 2014 Elsevier B.V. All rights reserved.

\section{HERMES experiment}

HERMES was a polarized deep inelastic scattering experiment. It used $27.5 \mathrm{GeV}$ electron/positron beam of DESY-HERA and internal gas targets of polarized and unpolarized hydrogen, deuterium etc. The purpose of the experiment was to study spin structure of the nucleon. At HERMES, the scattered electrons/positrons and also a part of produced hadrons were detected in coincidence. This is called semiinclusive measurement. The angular correlation among the directions of scattered electron, target spin and produced hadrons provided important information on spin structure of the nucleon. The particle identification of pion, kaon, proton and anti-proton in the momentum range of $2-15 \mathrm{GeV} / c$ was essential. The electron/positron beam was longitudinally polarized. The longitudinally polarized hydrogen and deuteron targets were used for double spin asymmetry $A_{L L}$ in deep inelastic scattering while transversely polarized hydrogen target was also used to study transverse spin structure.

\section{RICH of HERMES}

HERMES adopted Ring Imaging Cherenkov Detector (RICH) [1] for hadron identification as shown in Fig. 1. It was a dual-radiator $\mathrm{RICH}$. The radiators used were aerogel and $\mathrm{C}_{4} \mathrm{~F}_{10}$ gas. It was the first RICH where aerogel was used as radiator in an actual particle physics experiment with accelerators.

The refractive index of aerogel [2] was 1.0304 and that of $\mathrm{C}_{4} \mathrm{~F}_{10}$ was 1.00137. The refractive index of 1.03 corresponded to kaon momentum threshold of $2 \mathrm{GeV} / c$. The design of RICH is shown in Figs. 2 and 3. The wall of aerogel was made of 425 aerogel tiles. The size of each tile was
$114 \times 114 \times 11.3 \mathrm{~mm}^{3}$. The aerogel tiles were stacked in 5 layers, with 5 horizontal rows and 17 vertical columns. The Cherenkov photons from the radiators were reflected by a common mirror to a photon detector which consisted of 1934 phototubes of 3/4 in. diameter.

Two RICH's of the identical design were built and placed above and below the electron beamline.

Examples of Cherenkov rings are shown in Fig. 4. The Cherenkov angles are plotted as functions of momentum in Fig. 5 which show agreement with expected curves.

The main parameters on the performance of the RICH can be summarized as follows: The number of PMT hits increased as a function of particle momentum from the threshold to the saturated region of Cherenkov angle as expected. In the saturated region, the number of PMT hits was 10 on average for aerogel and 12 for $\mathrm{C}_{4} \mathrm{~F}_{10}$ gas [1].

The Cherenkov angle had distributions and its HWHM was about $0.01 \mathrm{rad}$ both for aerogel and $\mathrm{C}_{4} \mathrm{~F}_{10}$ gas in the saturated region of Cherenkov angle. The Cherenkov angle difference between pion and kaon was expected to be 0.03 rad for aerogel at $4 \mathrm{GeV} / c$ for example, but the pion ring from $\mathrm{C}_{4} \mathrm{~F}_{10}$ gas can also be used at $4 \mathrm{GeV} / c$. The pion identification efficiency was above $92 \%$ at low momenta, and above $98 \%$ for momenta larger than $4 \mathrm{GeV} / c$. Some particles were classified as 'non-identified', which can be regarded as the background in terms of particle identification. They were $5 \%$ in total above $2 \mathrm{GeV} / c$.

The efficiency of particle identification was measured using the decays of $\rho^{0}, \mathrm{~K}_{\mathrm{s}}, \phi$ and $\Lambda$ as tagged hadrons. It was confirmed that Monte Carlo study of RICH by HERMES was consistent with the results of the measurements. A correction for the efficiency was applied in data analysis. 


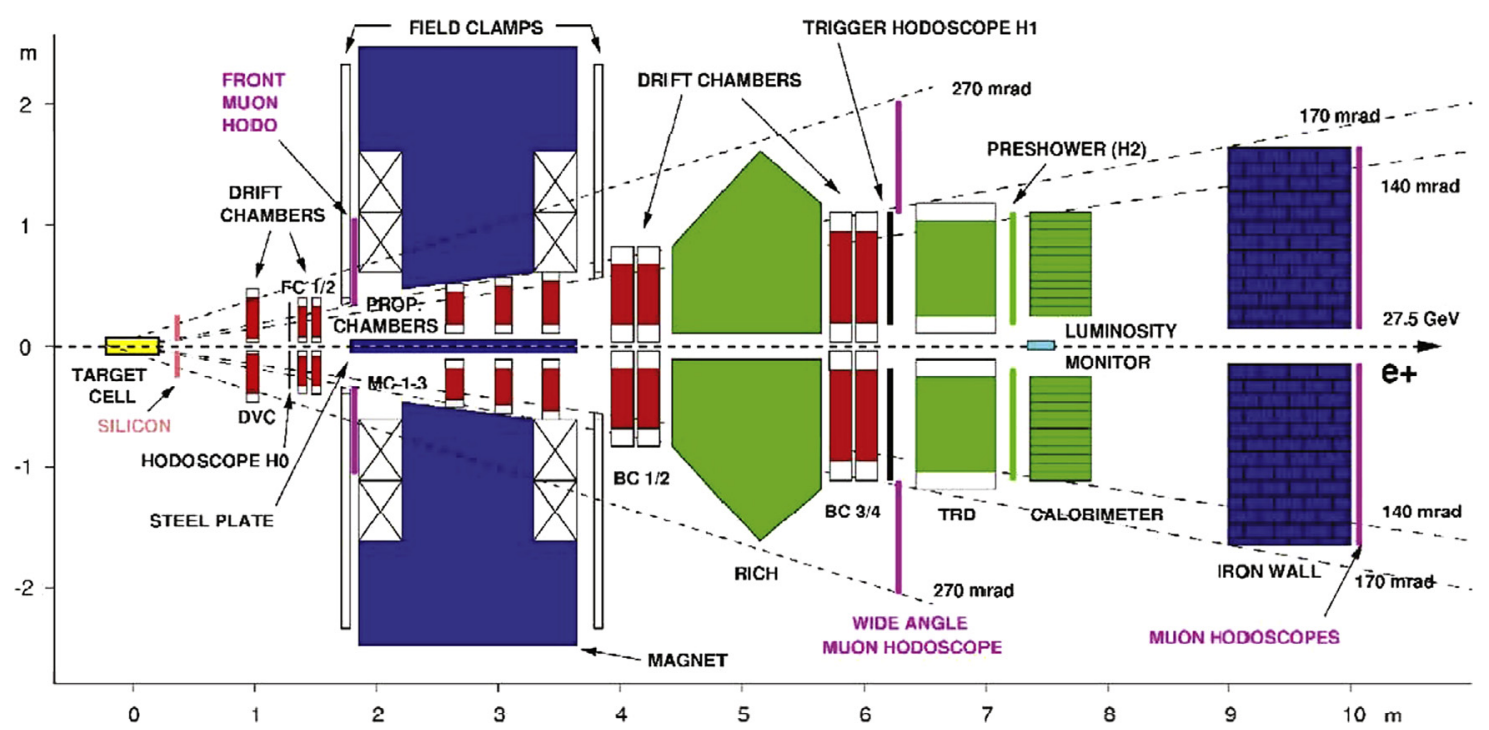

Fig. 1. The vertical cross-section of the HERMES spectrometer. The beam came from the left. RICH was located around the center in the figure.

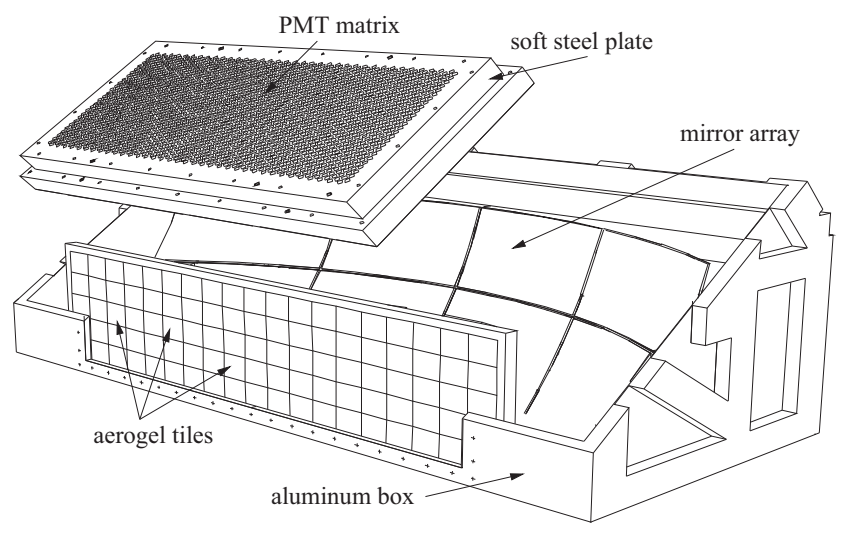

Fig. 2. The design of RICH. The volume between the aerogel wall and the mirror was filled with $\mathrm{C}_{4} \mathrm{~F}_{10}$ gas.

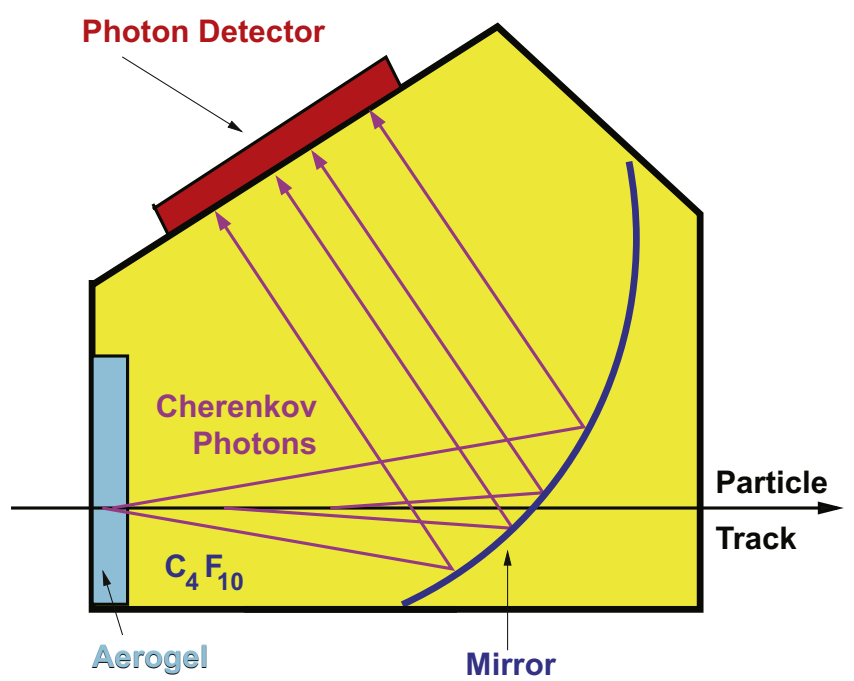

Fig. 3. The side view of the RICH. Cherenkov photons from aerogel and $\mathrm{C}_{4} \mathrm{~F}_{10}$ gas were reflected by a common mirror to the photon detector.

The data-taking with RICH continued for 10 years at HERMES and was completed in 2007. The RICH and its parts can therefore be used in other experiments in future. This way, the RICH of HERMES could further contribute to hadron identifications in particle physics experiments.
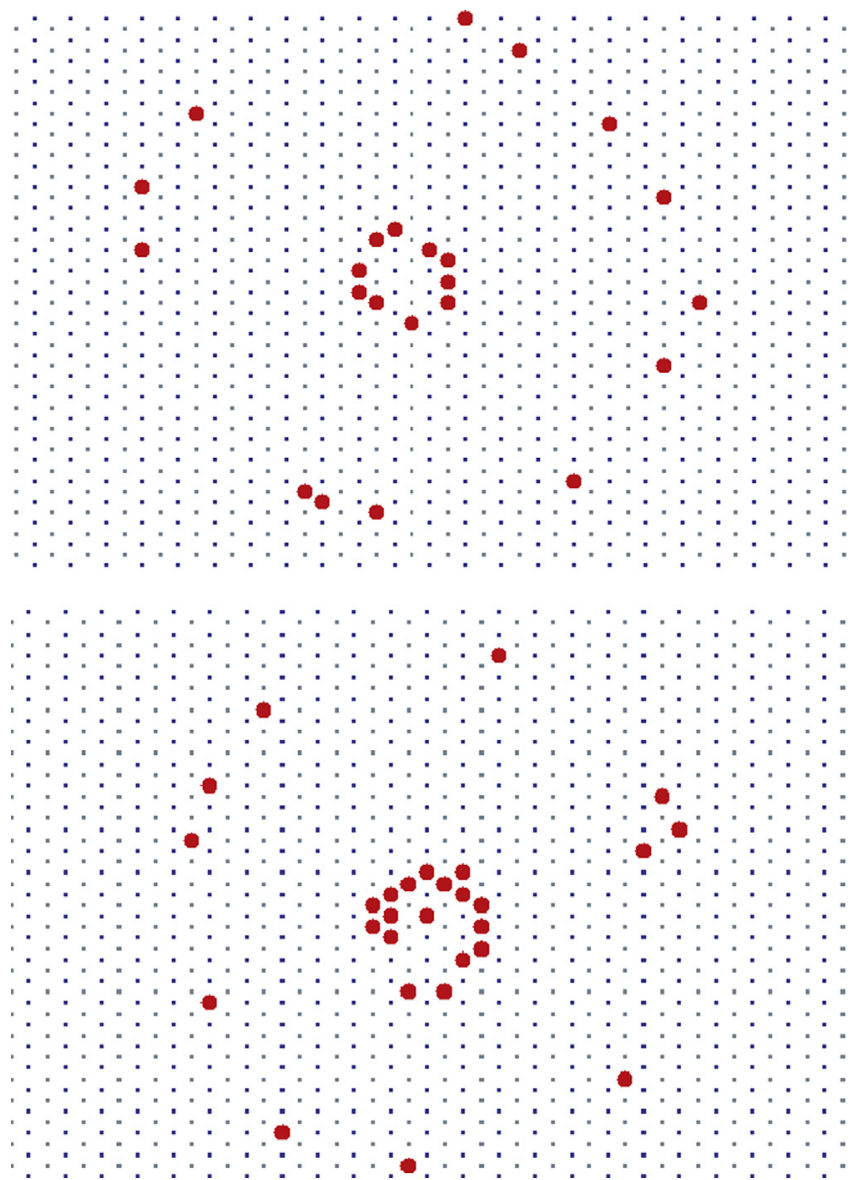

Fig. 4. Examples of detected Cherenkov photons by RICH of HERMES. The outer ring was due to aerogel while the inner ring was due to $\mathrm{C}_{4} \mathrm{~F}_{10}$ gas. Each small dot corresponds to the location of a phototube. Each large dot corresponds to the phototube which detected a photon.

\section{Physics results with RICH of HERMES}

Physics outputs from the data taken with the RICH at HERMES well indicate the good performance of the RICH. With the transversely polarized target, one can measure the angular correlation between the directions of the target spin, the scattered 


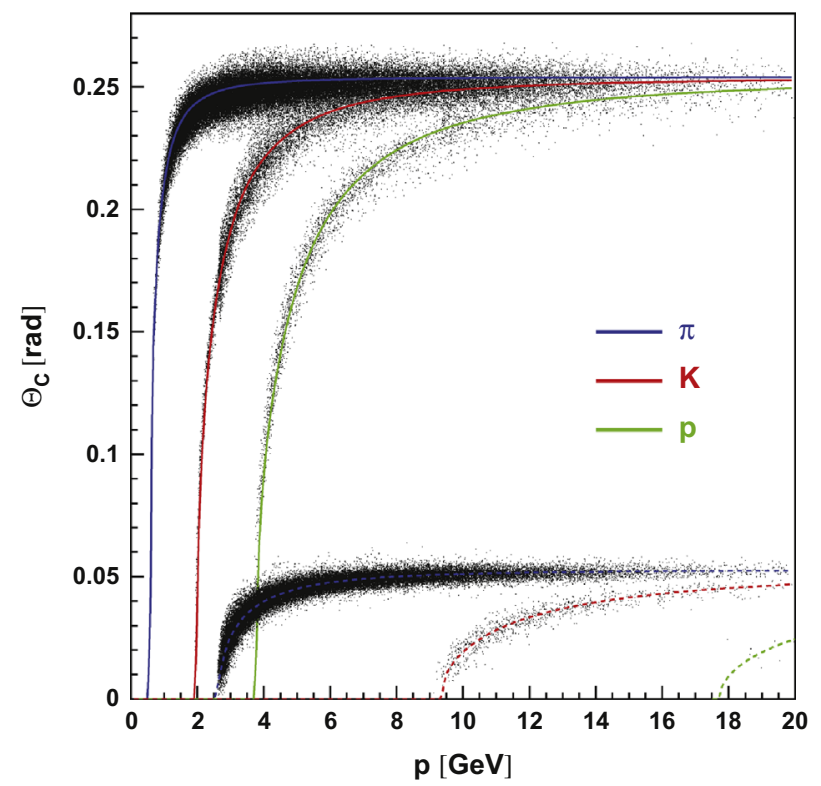

Fig. 5. The Cherenkov angle vs. hadron momentum. The curves show expected value. The dots show experimental data. The upper three curves are for pion, kaon and proton with aerogel while the lower three curves are for pion, kaon and proton with $\mathrm{C}_{4} \mathrm{~F}_{10}$ gas.

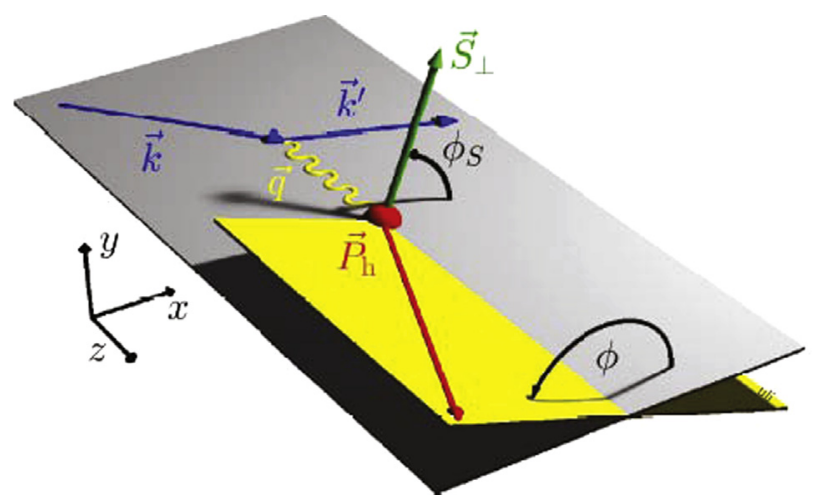

Fig. 6. The azimuthal angles $\phi$ of hadron production and $\phi_{S}$ of the target spin direction are defined in this figure around the virtual photon direction.

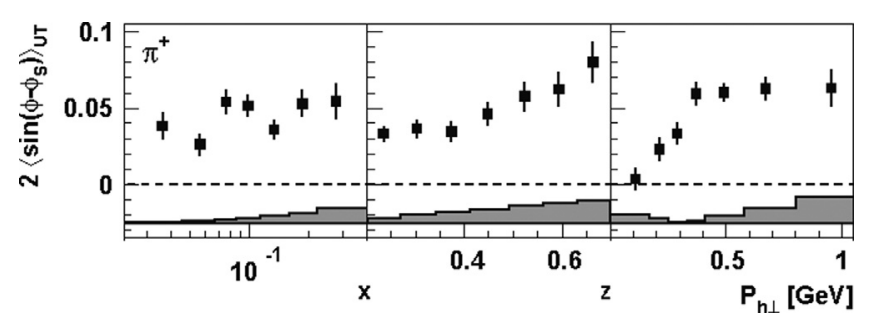

Fig. 7. Sivers asymmetry plotted against $x, z$ and $p_{h \perp}$ for $\pi^{+}$production [3].

electron and produced hadrons. The azimuthal angles $\phi$ and $\phi_{S}$ are the angles from the electron scattering plane as shown in Fig. $6 . \vec{k}$ and $\overrightarrow{k^{\prime}}$ are incident and scattered electron momenta. They determine the electron scattering plane. $\vec{q}$ is the momentum of exchanged virtual photon, $\overrightarrow{p_{h}}$ the momentum of the produced hadron.

The azimuthal asymmetry for $\pi^{+}$, for example, was non-zero [3] as shown in Fig. 7, giving an evidence for Sivers asymmetry. $x$ is Bjorken $x$, which is the fraction of the proton momentum carried by the struck quark in the reaction, $z$ is the ratio of energy of the

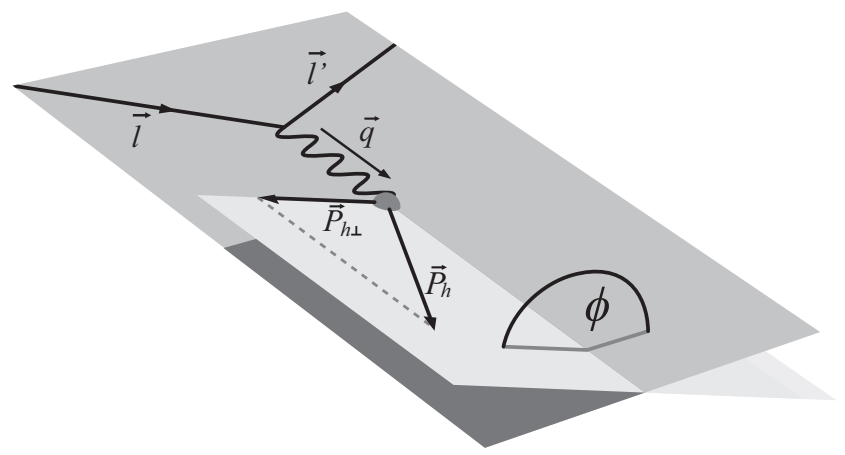

Fig. 8. The azimuthal angle $\phi$ between the hadron production plane and the electron scattering plane.
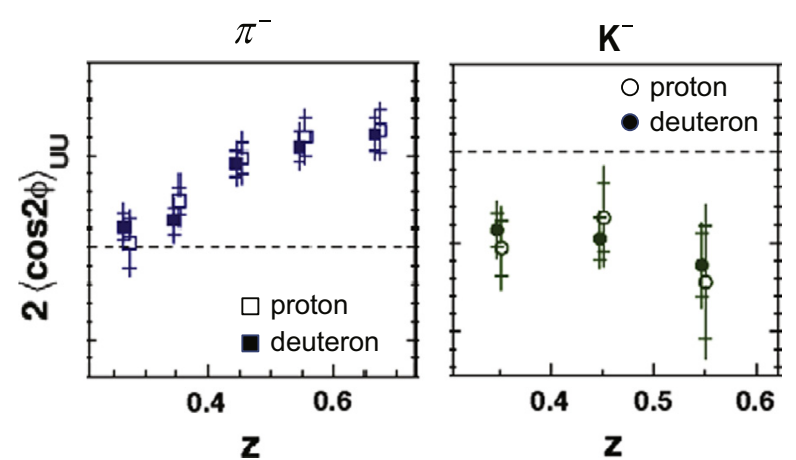

Fig. 9. The azimuthal asymmetry of hadron productions from unpolarized proton and deuteron targets [4]. The horizontal axis is $z$, which is the ratio of energy of the produced hadron to the exchanged virtual photon.

produced hadron to the exchanged virtual photon. $p_{h \perp}$ is the transverse hadron momentum.

Recently, a new type of azimuthal asymmetry was analyzed by HERMES. In the case of unpolarized target, the azimuthal angle between the electron scattering plane and hadron production plane can be defined as Fig. 8.

The azimuthal asymmetries of pions and kaons with unpolarized proton and deuteron targets were observed as shown in Fig. 9 [4]. The observed asymmetry gave a hint to study a correlation between spin and momentum of a quark in the unpolarized nucleon.

To summarize, the hadron identification by the RICH played an essential role in the series of data analysis of HERMES where hadrons were measured in coincidence with scattered electron. It brought us new insights into the structure of the nucleon both for longitudinal and transverse spin aspects.

\section{Acknowledgments}

We gratefully acknowledge the DESY management for its support, the staff at DESY and the collaborating institutions for their significant effort, and our national funding agencies for financial support.

\section{References}

[1] N. Akopov, et al., HERMES RICH Group, Nuclear Instruments and Methods in Physics Research Section A 479 (2002) 511.

[2] E. Aschenauer, et al., HERMES RICH Group, Nuclear Instruments and Methods in Physics Research Section A 440 (2000) 338.

[3] A. Airapetian, et al., HERMES, Physical Review Letters 103 (2009) 152002.

[4] A. Airapetian, et al., HERMES, Physical Review D 87 (2013) 012010. 